\title{
Estudo da condição de superfície em rodovias por meio do uso de aplicativo para smartphone
}

\author{
Lucas Cavalcante de Almeida ${ }^{1}$, Francisco Heber Lacerda de Oliveira ${ }^{2}$, Saulo Passos Ramos ${ }^{3}$
}

${ }^{1}$ Universidade Federal do Ceará, Departamento de Engenharia de Transportes, lucascavalcante@det.ufc.br

2Universidade Federal do Ceará, Departamento de Engenharia de Transportes, heber@det.ufc.br

3Universidade Federal do Ceará, Departamento de Engenharia de Transportes, saulo@det.ufc.br

\section{Recebido:}

18 de junho de 2017

Aceito para publicação:

9 de abril de 2018

Publicado:

31 de agosto de 2018

Editor de área:

Jorge Barbosa Soares

\section{Palavras-chaves:}

Smartphone

Textura,

Revestimento,

Defeitos.

\section{Keywords:}

Smartphone,

Texture,

Wearing Course,

Distress.

DOI:10.14295/transportes.v25i2.1406

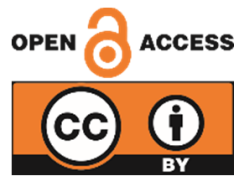

\begin{abstract}
RESUMO
Este artigo analisa as condições de superfície de vias pavimentadas com revestimento em Concreto Asfáltico, Tratamento Superficial e Concreto de Cimento Portland, além de uma via não pavimentada, por meio da utilização do aplicativo para smartphone denominado Roadroid. As características consideradas estão associadas à textura e aos defeitos de superfície. A análise é efetuada através de um gráfico BoxPlot comparativo e medidas de tendência central. Em relação às texturas, constata-se que revestimentos com texturas abertas apresentam maiores valores de irregularidade mesmo na ausência de defeitos. Verifica-se, também, que os trechos em estudo apresentam condição de superfície analisadas pelo aplicativo semelhante às fornecidas pelos órgãos rodoviários e que segmentos com alto coeficiente de variação apresentam quantidades significativas de defeitos, sobretudo remendos e buracos. Com esta análise, espera-se contribuir com dados mais confiáveis, obtidos por meio de smartphones, de modo a auxiliar a tomada de decisão dos órgãos gestores.
\end{abstract}

\begin{abstract}
This paper analyzes surface conditions of an Asphaltic Concrete paved road, Surface Treatment and Cement-Concrete, and also an unpaved road, using a smartphone application called Roadroid. The considered characteristics of surface conditions are associated with texture and surface distress. The analysis is performed utilizing a comparative BoxPlot chart and a central trend measures. In relation to the textures, it is verified that open macrotextures in wearing courses presents higher values of irregularity, even in the absence of distress. It is also observed that the surface conditions analyzed by the smartphone app are similar to the surface conditions provided by road agencies and those segments with high variation coefficient values, present a significant amount of distress, especially patches and potholes. With this analysis, it is expected to contribute with the most reliable data, obtained through the use of smartphones, in order to aid managing groups on decision-making.
\end{abstract}

\section{INTRODUÇÃO}

Em virtude do avanço tecnológico dos smartphones na última década, muitos aplicativos têm sido desenvolvidos para os mais diversos fins e ramos de atividades. No âmbito da Engenharia Civil e, mais especificamente, no da Engenharia Rodoviária, o uso dessas novas tecnologias tem proporcionado progressos significativos para as obras, bem como para os projetos e suas respectivas análises. Isso se torna mais evidente quando envolve trabalhos de coleta e de armazenamento de dados obtidos em campo.

No exterior, o uso dos aplicativos nos processos de avaliação in situ de pavimentos rodoviarios tem se tornado recorrente. No Brasil, tal prática é pouco aplicada. Países como a Suécia, a 
China e o Japão, por exemplo, fazem uso dos aplicativos para a obtenção de parâmetros de textura superficial dos revestimentos e de irregularidade longitudinal durante a passagem do tráfego. Desse modo, obtêm-se características relacionadas com a segurança e com o conforto ao rolamento.

Uma das vantagens percebidas na utilização dos aplicativos para a avaliação de pavimentos rodoviários é a realização de levantamentos mais rápidos, com maior desempenho e sem maiores interferências diretas e constantes do homem. Por conta disso, os resultados obtidos são, muitas vezes, mais acurados e com custos relativamente menores quando comparados aos métodos tradicionais. Há, ainda, o fato de que os resultados apresentados são facilmente visualizados e compreendidos pelos envolvidos na gestão dessas infraestruturas, o que facilita o processo de tomada de decisão.

Nesse contexto e considerando a carência de estudos e levantamentos de campo nas rodovias nacionais, o presente artigo tem o objetivo de realizar uma análise de dados obtidos por meio do aplicativo para smartphone denominado Roadroid e associar os resultados com características de textura e de conforto ao rolamento de diferentes tipos de revestimentos. 0 aplicativo em pauta foi desenvolvido por pesquisadores suecos, com larga utilização naquele país, e, para fins deste artigo, a aplicação foi feita em trechos de rodovias no Estado do Ceará.

\section{REVISÃO DE LITERATURA}

De acordo com Bisconsini (2016), alguns pesquisadores têm estudado o uso de smartphones para avaliação funcional dos pavimentos, principalmente na determinação da irregularidade longitudinal, devido sobretudo ao seu baixo custo, fácil operação e produtividade.

Ainda segundo Bisconsini (2016), a utilização de smartphones para a avaliação das irregularidades presentes nos revestimentos é baseada na utilização do sensor de movimento, também conhecido como acelerômetro, e um GPS (Global Positioning System), ambos já presentes nos aparelhos móveis. Assim, é possível captar os sinais de aceleração vertical decorrentes das irregularidades dos pavimentos, juntamente com a velocidade e a posição dos dados coletados.

González et al. (2008) começaram a utilizar acelerômetros embarcados em veículos para avaliar a condição de irregularidade da via. As conclusões das simulações realizadas nesses estudos foram que a irregularidade de uma via pode ser estimada a partir de dados de aceleração oriundos de sensores de smartphones.

Erikson et al. (2008) desenvolveram um sistema denominado Pothole Patrol, no qual o princípio básico era detectar buracos e remendos em rodovias a partir de dados de acelerômetros. Mohan et al. (2008), por sua vez, utilizaram diversos sensores de smartphones, tais como acelerômetros, microfone e GPS para monitorar o tráfego e a condição de rodovias.

De acordo com Douangphachanh e Oneyma (2014), ainda há poucos estudos que exploram diretamente o uso de smartphone ou sensores para estimar o valor do IRI (International Roughness Index - Índice Internacional de Irregularidade) dos pavimentos rodoviários. No entanto, já existem estudos para detecção de bumps e patologias, tais como buracos e remendos presentes em uma rodovia.

Apesar da afirmação de Douangphachanh e Oneyna (2014), esses autores descreveram as análises e relações entre dados obtidos por sensores de smartphones e o IRI. Dois experimentos, utilizando diversos tipos de smartphones e veículos foram realizados para coletar dados a partir de acelerômetros, giroscópios e GPS. Verificou-se que o IRI pode ser modelado aproximadamente como uma funcão linear das magnitudes de vibracão e velocidade média do deslocamento. 
Foi observado também que essa função linear depende das características dos veículos (sistema de amortecimento, massa, modelo), da velocidade de deslocamento, bem como do local de instalação do smartphone com suas respectivas configurações.

Wang e Guo (2016) descreveram um projeto piloto que permite a determinação de indicadores de condição da superfície da rodovia baseado em um aplicativo de smartphone. Este projeto teve o centro de coordenação na Bielorrússia, com apoio do Banco Mundial, para o qual foram coletados grandes volumes de dados, fornecendo ampla cobertura da rede rodoviária naquele país.

No experimento de Bisconsini (2016), foram medidos sinais de aceleração vertical por meio de um smartphone fixado ao painel de um veículo, que trafegou em diferentes velocidades em pavimentos rodoviários e aeroportuários. Com esses dados, calcularam-se os valores de RMSVA (Root Mean Square Vertical Acceleration - Média Quadrática da Aceleração Vertical) e os resultados foram confrontados com a irregularidade longitudinal (IRI) dos pavimentos. Obteve-se correlação positiva com coeficientes de correlação entre 0,97 e 0,99.

Outros pesquisadores, tais como Hanson e Cameron (2012), Alessandroni et al. (2014), Buttlar e Islam (2014) e Forslof (2015), também estudaram o uso de smartphones para avaliação da irregularidade longitudinal dos pavimentos, com resultados favoráveis à sua aplicação no segmento rodoviário.

\subsection{Conforto ao rolamento e defeitos de superfície}

De acordo com Silva (2005), as patologias funcionais são as que mais contribuem para afetar a segurança e as condições de dirigibilidade nos pavimentos. A rugosidade excessiva, o polimento dos agregados, o ruído e os defeitos de superfície são exemplos destas patologias, que podem ocorrer tanto em pavimentos rígidos, quanto em flexíveis. A rugosidade pode ser causada por saliências, que se devem a problemas durante a execução (vassouramento ou sobra de concreto descarregado sobre concreto endurecido) ou inadequações dos serviços de compactação. 0 polimento da superfície pode ser devido à disposição de lona para proteger o concreto da chuva; isto pode causar problema de segurança se não for corrigido. Os defeitos de superfície podem ser causados, também, pela retração plástica do concreto, delaminação, erosão e marcas de pneus, além de ausência de manutenção.

Ainda de acordo com Silva (2005), as patologias em pavimentos flexíveis podem ser classificadas como: deformações de superfície (corrugação e afundamentos), defeitos de superfície (exsudação de asfalto e desgaste), panela, escorregamento do revestimento asfáltico e trincas. Para CNT (2016), os defeitos de superfície fazem com que o pavimento apresente sinais de desgaste, com efeito de desagregação progressiva do agregado da massa asfáltica e aspereza superficial no revestimento, comprometendo a segurança e o conforto ao rolamento. Esse desgaste superficial é resultante da associação do tráfego com o intemperismo, e o arrancamento progressivo dos agregados é um estágio avançado do desgaste superficial.

Oliveira et al. (2016), em seus estudos iniciais sobre o comportamento da aderência e do conforto ao rolamento em pavimentos revestidos por Concreto Asfáltico, concluíram que, em condições funcionais adequadas, quanto mais rugosa a superfície do revestimento, melhores são as condições de drenagem superficial e maiores são os valores de aderência atingidos pelo contato pneu-pavimento. Entretanto, no que diz respeito ao conforto e à qualidade de rolamento, naquelas condições de atrito e mesmo o revestimento não apresentando defeitos de superfície, os valores de irregularidade longitudinal encontrados foram inadequados. 
Para Bernucci et al. (2008), a irregularidade interfere na dinâmica dos veículos e no contato do veículo com o pavimento, afetando a estabilidade direcional e a aderência em pistas molhadas. Além disso, Bernucci et al. (2008) afirmam ainda que texturas de pavimentos asfálticos classificadas de média a aberta são recomendadas, enquanto texturas muito abertas causam desgaste excessivo nos pneus e tendência a maior ruído ao rolamento.

\section{2. $O$ aplicativo Roadroid}

O aplicativo utilizado para realização da coleta de dados neste trabalho foi o Roadroid. Esse aplicativo desenvolvido por Forslof (2015), na Suécia, começou a ser elaborado no ano de 2002 e possui como princípio a análise de dados do acelerômetro, a velocidade de deslocamento do veículo e o intervalo de amostragem. Outros aplicativos estão disponíveis para uso e avaliação dos dados coletados, tais como TotalPave e RoadBump, no entanto foi preferível o Roadroid devido à sua fácil e direta interface com o usuário.

Em meados de 2011, os desenvolvedores do Roadroid começaram a coletar dados em diferentes tipos de rodovias e de veículos passando por obstáculos distintos (Figura 1).

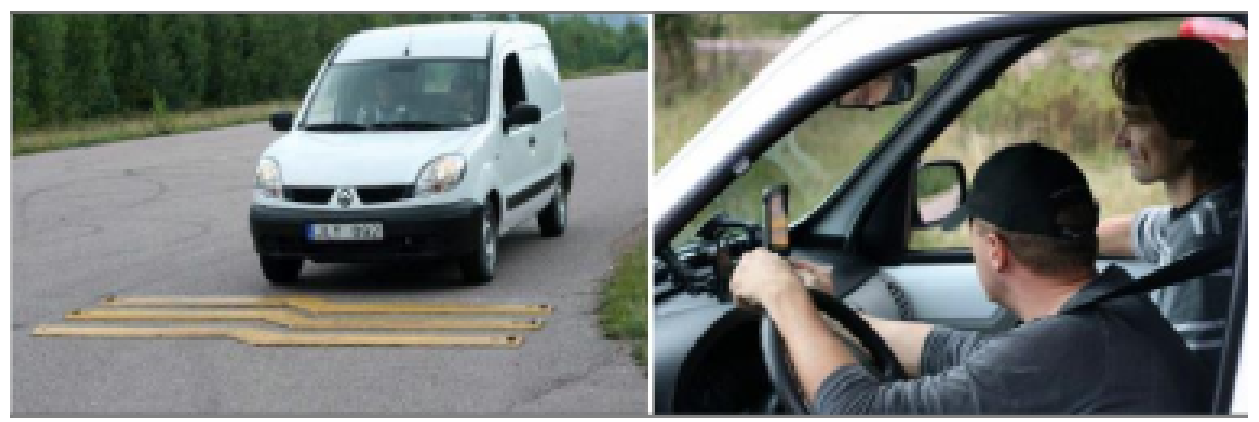

Figura 1: Obstáculos para estudo e fixação do smartphone no veículo (Forslof, 2015)

De acordo com Hirpahuanca (2016), os obstáculos foram percorridos por diferentes tipos de veículos, desde carros de passeio até $4 \mathrm{WD}$, com seis diferentes tipos de velocidades $(20,40,60$, 80, 100 e 120km/h). Ainda de acordo com Hirpahuanca (2016), estes experimentos realizados durante o ano de 2011 podem ser utilizados como dados confiáveis. A análise dos dados permitiu fazer as seguintes observações:

a) Foram verificadas diferenças entre os tipos de veículos, especialmente quando percorriam as vias com baixas velocidades. No entanto, verificou-se que em um intervalo de velocidade entre 40 e $80 \mathrm{~km} / \mathrm{h}$, estas diferenças tendem a diminuir;

b) Foi encontrado um modelo que permite calcular a influência da velocidade em três tipos de veículos (pequeno, médio e grande porte). Também foram verificadas as diferenças entre dispositivos distintos, especialmente em relação à taxa de aquisição de dados e a sensibilidade dos acelerômetros. Foi realizado um processo de calibração que permitiu padronizar os parâmetros de cada dispositivo de coleta.

c) Verificou-se também que, para ter uma confiabilidade dos dados, é preciso que se tenha um suporte ou uma base rígida e estável na qual o smartphone deve ser disposto.

Um dos dados de saída do Roadroid, é o eIRI (IRI estimado). Esse foi o parâmetro utilizado nesta pesquisa. Para obter esse dado, Forslof (2015) realizou medições de IRI com equipamentos de classe 1 (alta precisão) a cada 20 metros e comparou com os dados de eIRI estabelecendo um fator de correlação de 75\%. Essa correlação também foi realizada por diferentes instituições 
em todo o mundo, como o Banco Mundial, as Nações Unidas, universidades e empresas de engenharia ao longo do ano de 2014. Resultados mostraram que em sua maior parte foram alcançadas correlações de até $80 \%$ ente o IRI e o eIRI.

Assim, de acordo com Forslof (2015), em estudos realizados na Universidade de Auckland em 2013, o Roadroid respondeu de forma similar aos sistemas utilizados pelo setor rodoviário de Auckland, atingindo 81\% de correlação com os sistemas de medições a laser.

0 eIRI é calculado com base nos valores de RMS (Root Mean Square) provenientes do acelerômetro presente no smartphone e, segundo Forslof (2014), possui correlação tanto com a textura, quanto com a irregularidade longitudinal (Figura 2). Neste artigo, não foi possível distinguir as contribuições específicas de valores de eIRI para a textura e para a irregularidade longitudinal, pois o desenvolvedor do aplicativo não disponibilizou a equação de cálculo do eIRI.

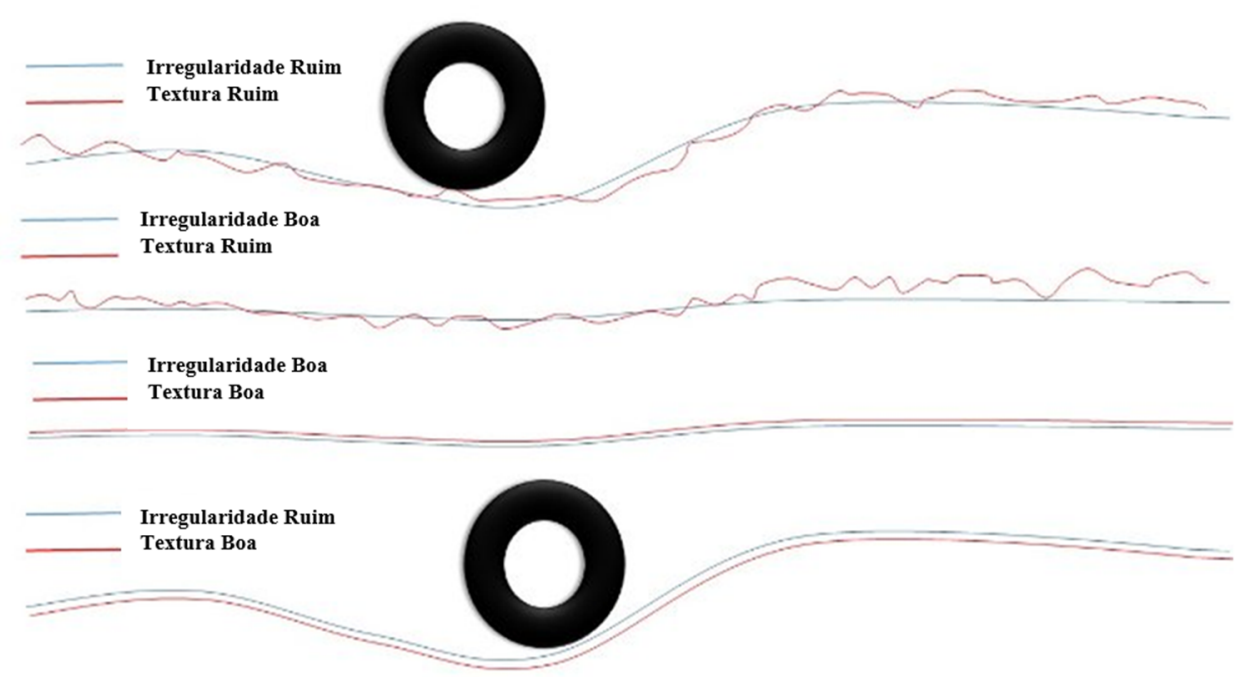

Figura 2: Relação entre textura e irregularidade (Forslof, 2014)

Ainda com base na Figura 2 e a partir dos estudos de Forslof (2014 e 2015), no que diz respeito às relações apresentadas, as classificações boa e ruim referem-se quanto à textura ser fechada ou aberta, respectivamente, e à presença de defeitos (tais como remendos e buracos) que possam gerar menor ou maior irregularidade ao rolamento dos veículos.

Por sua vez, Aps (2006) afirma existir sobreposição de valores dos intervalos de dimensões de ordem vertical para diferentes domínios de textura e irregularidade. 0 domínio da macrotextura possui intervalos de dimensões variando entre $0,2 \mathrm{~mm}$ a $10 \mathrm{~mm}$, enquanto que para a irregularidade longitudinal esse intervalo é de 1,0 $\mathrm{mm}$ a $50 \mathrm{~mm}$. Essa sobreposição pode justificar a contribuição dos valores de eIRI contabilizados para a textura e para a irregularidade longitudinal.

Os autores desta pesquisa entendem que, possivelmente, o eIRI guarda alguma relação com o perfil longitudinal, haja vista que, pelos estudos realizados, quanto mais irregular o perfil, maiores tendem a ser os valores da aceleração vertical, consequentemente maior o valor de RMS. 0 desenvolvedor do aplicativo, em seus estudos (Forslof, 2014 e 2015), não é específico quanto à relação, direta ou indireta, do eIRI com o perfil longitudinal, mencionando apenas a questão da textura. Diante disso, acredita-se que a megatextura é a de maior influência, pois o domínio da dimensão vertical diz respeito à amplitude das classes de textura, conforme descrito por Aps (2006). 
Além dos valores de eIRI, uma ferramenta visualizadora de mapas baseada em HTML5 apresenta os dados da condição da via, conforme Figura 3.

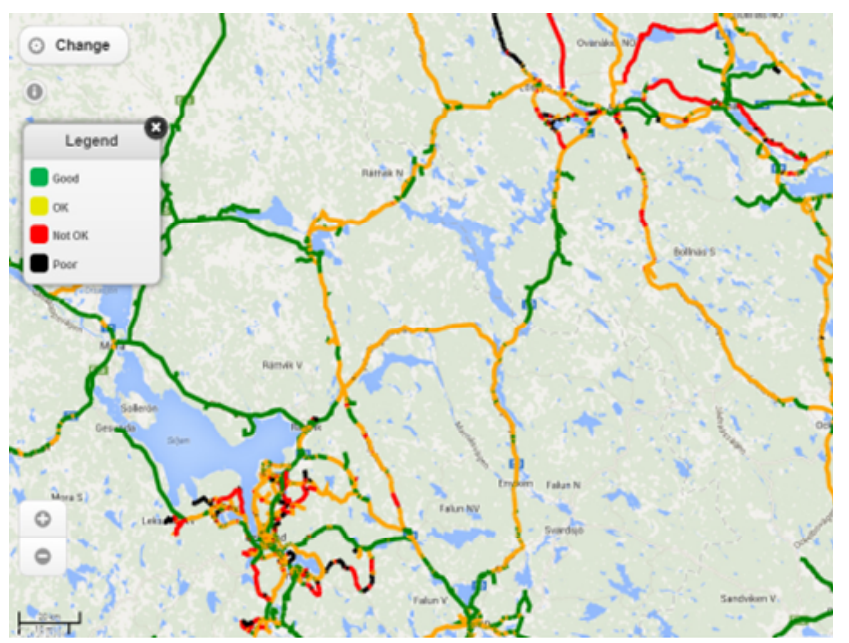

Figura 3: Mapa de classificação do elRI gerado pelo Roadroid (Forslof, 2015)

O Roadroid indica que a condição da via é dividida em quatro diferentes níveis de qualidade funcional e cores, de acordo com os valores de eIRI: verde para boa $(0,0$ a 2,2), amarelo para regular $(2,2$ a 3,8$)$, vermelho para ruim $(3,8$ a 5,4$)$ e preto para péssimo $(>5,4)$. Essa escala foi desenvolvida por Forslof (2015), possuindo correlação qualitativa com o IRI, no entanto, é adimensional.

\section{MÉTODOS DE OBTENÇÃO DOS DADOS}

Foram realizados levantamentos com utilização do Roadroid, a partir de único smartphone cujo aplicativo fornece configurações para este modelo. 0 aparelho foi fixado no para-brisa de um veículo de passeio, que trafegou com as seguintes velocidades médias: $80 \mathrm{~km} / \mathrm{h}$ para trechos revestidos por Concreto Asfáltico (CA) e Tratamento Superficial (TS); velocidade média de $55 \mathrm{~km} / \mathrm{h}$ para os trechos com revestimento Concreto de Cimento Portland (CCP); e velocidade média de $33 \mathrm{~km} / \mathrm{h}$ na via não pavimentada (NP).

A determinação da velocidade do experimento foi em função da condição da rodovia e da velocidade máxima permitida. Todos os trechos pertencem a rodovias estaduais (CE) ou federais (BR) do Estado do Ceará e foram analisados durante o período diurno, de modo a facilitar a inspeção visual. 0 equipamento e o veículo utilizado são mostrados na Figura 4.

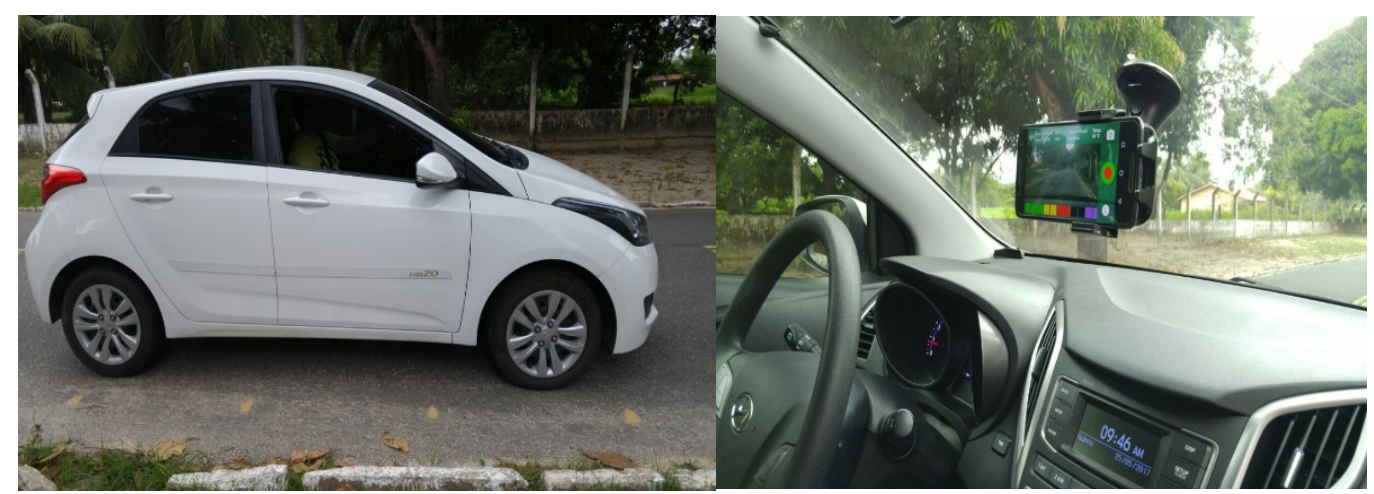

Figura 4: Veículo e equipamento utilizados no experimento 
Os dados de eIRI, obtidos com a utilização do Roadroid, foram tratados estatisticamente utilizando a ferramenta BoxPlot. As medidas de tendência central foram determinadas por meio do software Minitab®, de modo a comparar os trechos analisados. Finalmente, a análise foi realizada por meio de um BoxPlot comparativo para revelar semelhanças ou diferenças entre os conjuntos de dados obtidos.

\subsection{Descrição dos Trechos Analisados}

Os trechos analisados estão localizados na Região Metropolitana de Fortaleza (RMF), Sertão Central do estado do Ceará e na cidade de Fortaleza, totalizando cerca de $415 \mathrm{~km}$. A seguir estão indicados os segmentos específicos das rodovias percorridas com as respectivas siglas entre parênteses:

a) Na RMF: BR-116 entre as cidades de Chorozinho a Itaitinga (CHZ-ITA);

b) No Sertão Central: entre as cidades de Quixeramobim a Madalena (QXB-MAD), cidade de Choró à BR-020 (CHR-BR020), CE-060 a Choró (CE060-CHR), Quixadá via CE-060 (QXDCE060), BR-122 entre Quixadá e Pirangi (QXD-PIR) e entre Pirangi e Chorozinho (PIRCHZ), BR-020 entre Canindé e Fortaleza (CAN-FOR), e trecho de via não pavimentada localizada na cidade de Madalena (TEOTONIO);

c) Em Fortaleza: Avenida Dioguinho (DIOG) e o Quarto Anel Viário de Fortaleza, que contemplam pavimentos rígido $\mathrm{AV}(\mathrm{CCP})$ e flexível $\mathrm{AV}(\mathrm{CA})$.

\subsection{Verificação dos Dados Coletados e Limitações do Dispositivo}

Para a comparação qualitativa dos dados de eIRI obtidos pelo Roadroid, foi utilizada a especificação de serviço DNER (1986), que recomenda o método de Nível e Mira para calibrar sistemas medidores de irregularidade tipo resposta. 0 trecho utilizado na verificação possui extensão de 320 m localizado no campus da Universidade Federal do Ceará com superfície revestida por TS. A Figura 5 apresenta vista geral e detalhada da superfície do trecho.

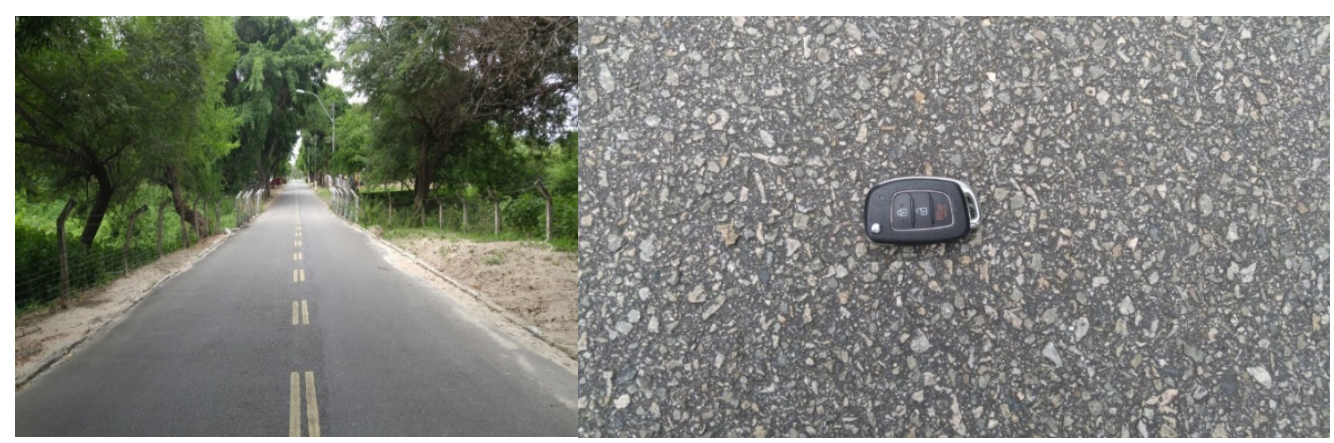

Figura 5. Vista geral do trecho para calibração e detalhe do revestimento.

Para correlacionar valores de IRI obtidos pelo método de Nível e Mira com dados de eIRI, discretizou-se os valores de IRI a cada $10 \mathrm{~m}$, assim como os dados de eIRI obtidos com velocidade de operação de $50 \mathrm{~km} / \mathrm{h}$, obtendo-se, portanto, 32 pontos. Como resultado, verificou-se que $63 \%$ da variável dependente (IRI) consegue ser explicada pelos regressores presentes no modelo (eIRI) conforme evidencia a Figura 6.

Quanto às limitações do dispositivo, por ser um equipamento que se baseia na resposta do sistema de suspensão dos veículos, obtendo assim valores de IRI de forma indireta, o Roadroid 
é classificado como um equipamento do tipo resposta. A instalação feita no para-brisa do veículo visa facilitar a interface com o usuário. Contudo, pode informar resultados imprecisos quando o dispositivo não está corretamente calibrado, haja vista que quanto mais rígido for o sistema de amortecimento, melhores são as respostas para modelagem do sistema.

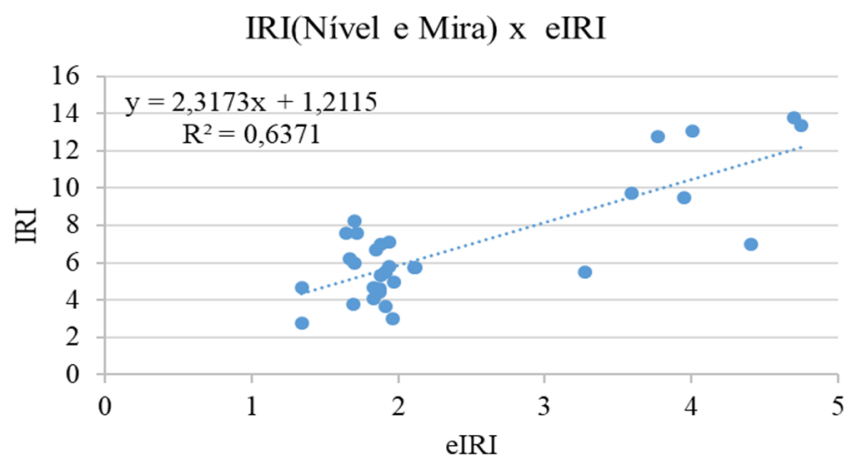

Figura 6. IRI (Nível e Mira) x elRI

Conforme Douangphachanh e Oneyma (2014), dentre as vantagens desse tipo de sistema podem-se mencionar: custo relativamente baixo, rápido levantamento e acurácia moderada, alta performance e adequação entre os instrumentos de medição de irregularidade. Por sua vez, dentre as desvantagens estão o fato de se despender alguns custos para o seu desenvolvimento e envolver trabalho exaustivo para calibração e configurações iniciais.

\section{ANÁLISE E DISCUSSÃO DOS RESULTADOS}

Serão apresentados o BoxPlot comparativos dos trechos analisados, assim como a análise dos segmentos, dados estatísticos e condição de superfície. No gráfico da Figura 7, os dados obtidos em campo estão divididos por tipo de revestimento: TS, CA, CCP e NP. A Tabela 1 complementa a análise com dados estatísticos e condição da superfície conforme dados dos órgãos responsáveis pela gestão da rodovia.

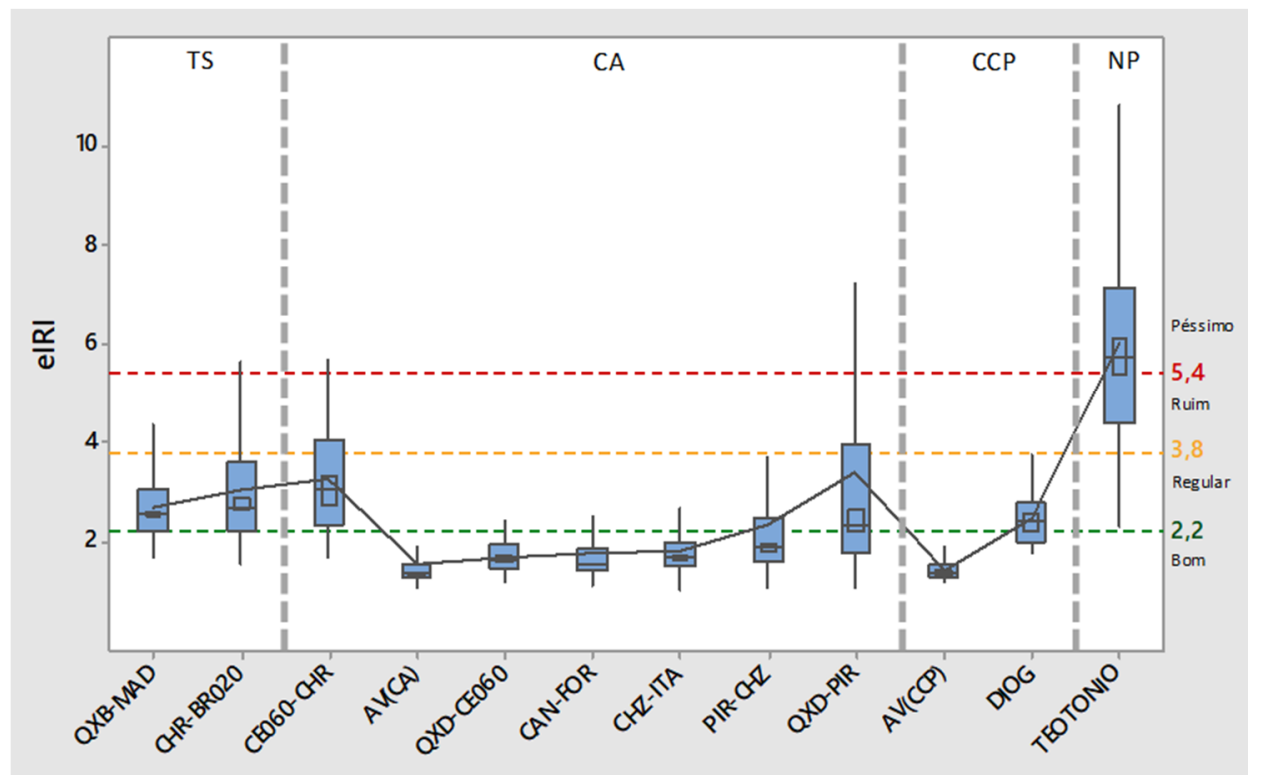

Figura 7. BoxPlot de Revestimentos (TS, CA e CCP) e Via Não Pavimentada (NP) 
Tabela 1: Descrição dos trechos, dados estatísticos e condição da superfície

\begin{tabular}{l|l|l|l|l|l}
\hline Sigla & $\begin{array}{l}\text { Tipo de Revestimento } \\
\text { / Superfície }\end{array}$ & $\begin{array}{l}\text { Extensão Pesquisada } \\
\mathbf{( K m )}\end{array}$ & elRI Médio & $\begin{array}{l}\text { Coeficiente } \\
\text { de Variação } \\
\text { (\%) }\end{array}$ & $\begin{array}{l}\text { Condição da } \\
\text { Superfície } \\
\text { (DER-CE/DNIT) }\end{array}$ \\
\hline QXB-MAD & & 59,6 & 2,70 & 24,88 & Bom \\
CHR-BR020 & TS & 42,7 & 3,07 & 38,14 & Regular \\
CEO60-CHR & & 12,1 & 3,28 & 34,34 & Regular \\
AV(CAUQ) & & 20,1 & 1,54 & 38,23 & - \\
QXD-CEO60 & & 11 & 1,70 & 17,20 & Bom \\
CAN-FOR & CA & 114,2 & 1,78 & 36,59 & Regular a Bom \\
CHZ-ITA & & 50,5 & 1,84 & 30,76 & Regular a Bom \\
PIR-CHZ & & 46,2 & 2,34 & 64,89 & Regular a Bom \\
QXD-PIR & & 45,1 & 3,40 & 80,73 & Regular \\
AV(RÍG) & CCP & 5,9 & 1,45 & 13,36 & - \\
DIOG & NP & 5,5 & 2,47 & 19,98 & - \\
TEOTONIO & & 1,8 & 6,03 & 39,42 & - \\
\hline
\end{tabular}

\subsection{Trechos com Revestimento em TS}

Os trechos que possuem revestimento do tipo TS tendem a apresentar maiores valores de eIRI médio, uma vez que o eIRI obtém valores de irregularidade e textura (possivelmente megatextura) concomitantemente. Esses trechos possuem como característica, maior exposição do agregado e menor aplicação de taxa de ligante, quando comparado ao CA. Outra característica é que os revestimentos do tipo TS não são executados com equipamentos do tipo vibroacabadoras, o que poderia reduzir as imperfeições resultantes da base, contribuindo para o decréscimo no valor do IRI.

O trecho QXB-MAD, apesar de apresentar boas condições de rolamento, segundo DER-CE (2017), e ser um trecho relativamente novo (construído em 2012), conforme Casa Civil (2012), apresentou também o valor de eIRI médio com classificação "Regular" pelo Roadroid, sugerindo um relativo desconforto ao usuário e uma textura mais aberta conforme pode ser observado na Figura 8.

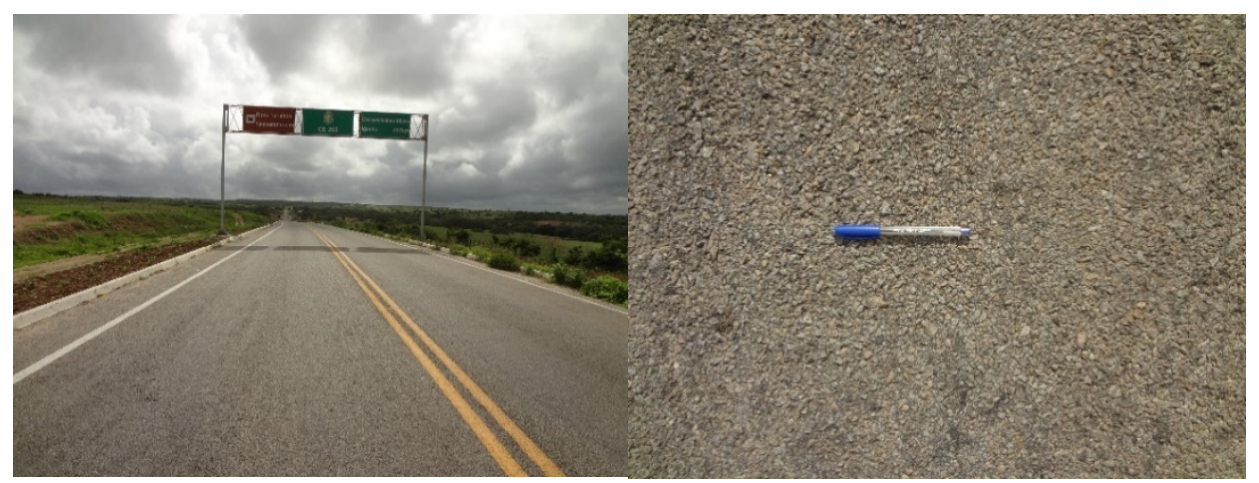

Figura 8. Vista geral do trecho e detalhe da textura (QXB-MAD)

Em relação aos outros trechos revestidos por TS, tais como CHR-BR020 e CE060-CHR, a tendência de comportamento em relação aos valores de eIRI é semelhante ao trecho QXB-MAD. No entanto, devido à presença de defeitos, tais como remendos, buracos, oxidações e desagregações, conforme evidenciado na Figura 9, os valores de eIRI ficaram mais dispersos e com média maior quando comparados ao trecho QXB-MAD. A despeito da presença dessas patologias, a classificação obtida pelo Roadroid também foi de "Regular" para ambos os trechos, apesar de 
nos trechos CHR-BR020 e CE060-CHR existirem valores considerados ruins. A condição de superfície obtida pelo Roadroid coincidiu com a classificação apresentada por DER-CE (2017) e pela avaliação feita pelos autores por ocasião dos levantamentos dos dados nos trechos.


Figura 9. Vista geral do trecho, detalhe da textura, presença de trincas e remendos (CHR-BR020)
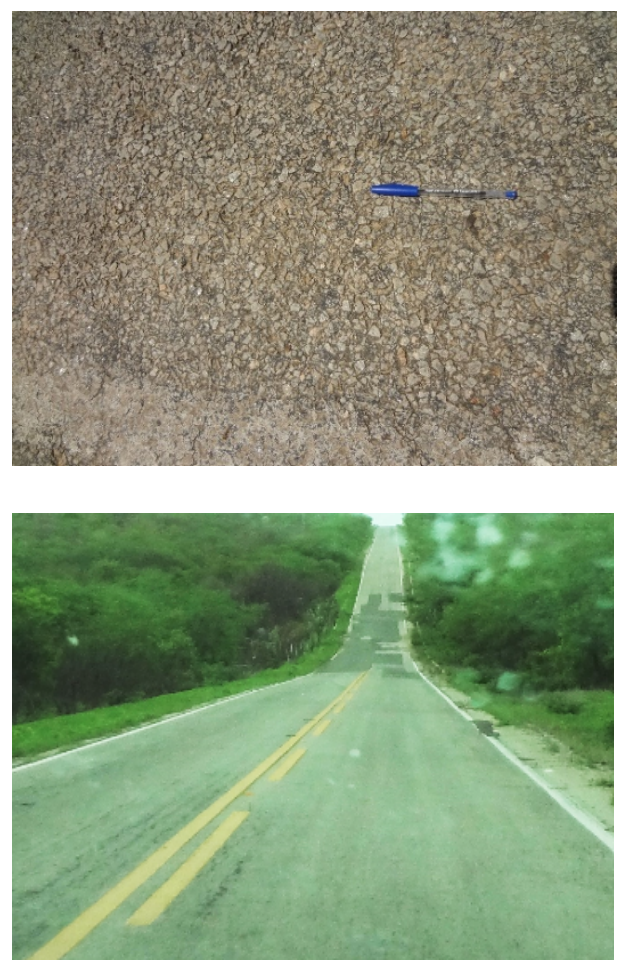

\subsection{Trechos com Revestimentos em CA}

Os trechos revestidos por CA, em condições funcionais normais, tendem a apresentar textura classificada de média a fechada, devido à taxa de aplicação do ligante ser maior que a aplicada em um TS e possuir granulometria bem graduada e contínua, além de menor exposição do agregado. Essa característica é confirmada em DNIT (2013), que indica as classes de textura para TS variando de média a aberta, enquanto que para os CA variam de média a fechada.

Diante do exposto, espera-se que valores de eIRI médio estejam situados dentro da classificação do tipo "Bom" pelo Roadroid. No entanto, o trecho analisado QXD-PIR apresentou grande dispersão dos dados, atingindo coeficiente de variação de $80,73 \%$, o maior de todos os trechos analisados, e valores de eIRI médio acima do esperado.

Essa dispersão e anormalidade dos resultados, possivelmente, devem-se ao fato de que, no trecho em questão, existem segmentos com grande quantidade de remendos, buracos e trincas, conforme observado na Figura 10. Esses defeitos contribuem para a majoração dos valores de eIRI médio, sugerindo que o trecho analisado apresenta maior desconforto aos usuários. De acordo com DNIT (2016), a condição da superfície desse trecho é definida como em condições regulares de tráfego, confirmando a classificação oriunda do Roadroid, fornecida também como de "Regular" ao conforto ao rolamento.

Outro trecho que apresentou alto coeficiente de variação $(64,89 \%)$ foi PIR-CHZ. Este segmento apresenta formação de trilha de roda devido ao tráfego pesado atuante e presença de remendos, dentre outros defeitos de superfície e estruturais (afundamentos).

Para os outros trechos revestidos por CA, os valores de eIRI médio apresentaram a classificação "Bom". A condição da superfície fornecida por DER-CE (2017) e DNIT (2016) foi de regular a bom, pois possuem uma textura fechada e existem poucos defeitos quando comparado aos trechos anteriores, QXD-PIR e PIR-CHZ. 


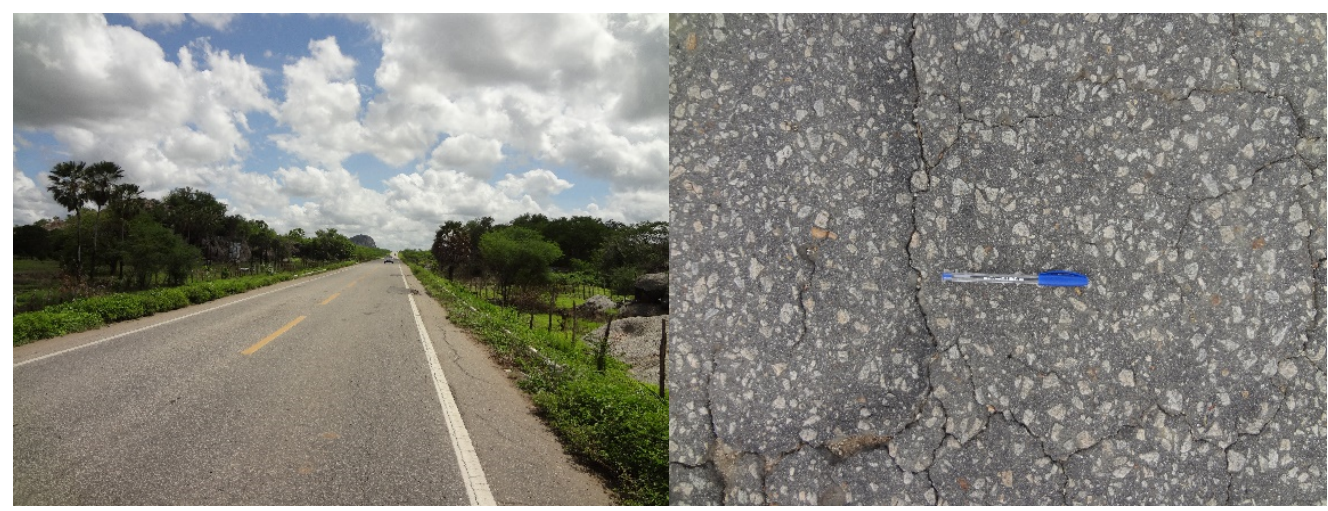

Figura 10: Vista geral do trecho e detalhe da textura (QXD-PIR)

\subsection{Trechos com Revestimento em Concreto de Cimento Portland}

Para os pavimentos de CCP foram analisados dois trechos, AV(CCP) e DIOG. Devido aos revestimentos possuírem as mesmas características de textura superficial e a mesma idade, esperava-se que o comportamento do gráfico apresentasse a distribuição ou dispersão semelhante dos dados de eIRI. No entanto, observou-se que o trecho DIOG apresentou maior dispersão dos dados e média de eIRI quando comparado ao $A V(C C P)$.

Por meio da observação feita in loco, foi possível constatar que essa dispersão dos dados se deve, possivelmente, à execução das placas de CCP e ao processo de texturização manual aplicado no trecho DIOG. Este mesmo segmento apresenta diversas ranhuras transversais deixando a superfície bastante rugosa, diferentemente do que ocorre no trecho AV(CCP), segmento no qual foi utilizado processo de texturização mecânico, deixando a superfície mais uniforme e menos rugosa, conforme mostra Figura 11. Este trecho apresentou o menor valor de eIRI médio $(1,45)$ e menor coeficiente de variação $(13,36 \%)$ dentre todos os segmentos analisados, apresentando a classificação "Bom" de conforto ao rolamento.

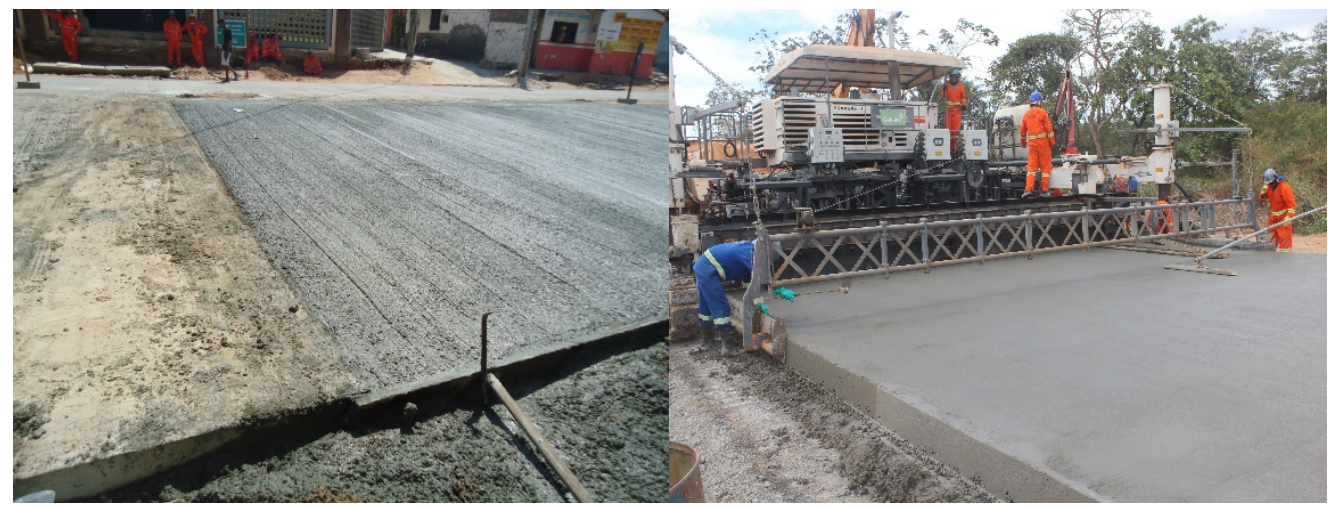

Figura 11. Superfícies texturizadas: trecho DIOG à esquerda e AV(RÍG) à direita

\subsection{Trecho de Superfície Não Pavimentada}

0 trecho com superfície NP (TEOTONIO) apresentou o maior eIRI médio $(6,03)$. Essa condição é esperada quando comparada a segmentos revestidos. Isso deve-se ao fato de que, nesse tipo de via, não existe qualquer revestimento e o terreno natural é a própria superfície de rolamento dos veículos (Figura 12). A regularização superficial da camada de rolamento é executada, geralmente, apenas por motoniveladora. Soma-se a isso, o fato de que esse trecho é utilizado como escoamento de produção agropecuária de uma fazenda da região com veículos pesados, contribuindo para o desgaste da superfície e consequente aumento do desconforto, contribuindo para classificação do tipo "Péssimo". 


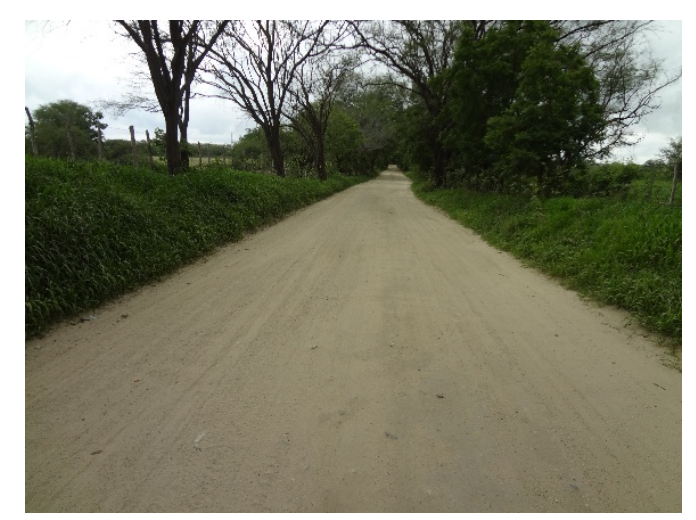

Figura 12. Via Não Pavimentada - Trecho TEOTONIO

\section{CONCLUSÕES}

As análises preliminares, obtidas por meio dos dados deste artigo, indicam que as classificações de condição ao rolamento fornecidas pelos órgãos rodoviários (DER e DNIT) e pelo Roadroid são condizentes entre si. Com o advento da tecnologia embarcada em dispositivos móveis, cada vez mais utilizam-se smartphones para a aquisição de vários tipos de dados. Tal condição pode auxiliar os órgãos gestores nas tomadas de decisões.

Os defeitos superficiais constatados nos levantamentos desta pesquisa, sobretudo remendos e trincamentos, proporcionaram maiores valores de eIRI médio. Verificou-se que nos trechos executados em TS, mas sem a presença de defeitos superficiais relevantes, o eIRI calculado foi relativamente baixo (da ordem de 2,7). Por sua vez, observou-se que trechos em TS com muitos remendos, e classificado como regular, apresentaram eIRI calculado acima de 3,0, e, assim, todos os trechos que apresentaram valores de eIRI acima de 3,0 tiveram classificação regular. A afirmação anterior é feita sem levar em consideração que defeitos, geralmente de origem construtiva e mais presentes em TS do que em CA, como as ondulações, também tendem a amplificar a irregularidade longitudinal. Conclui-se, portanto, que tais condições remetem a irregularidades que são influenciadas principalmente pela megatextura.

Para pavimentos de CCP, observou-se que a texturização, quando executada por processos automatizados, apresenta menores valores de eIRI médio, o que contribui para melhoria do conforto ao rolamento quando comparada à texturização feita manualmente. Na análise da via NP, apesar de não ser verificado aspecto da textura propriamente, foi possível observar o maior valor de eIRI devido à presença de imperfeições no corpo estradal natural.

A exposição do agregado, apesar de não ser caracterizada como um defeito de superfície, também pode ser constatada na análise dos dados. Essa característica pode servir como um dado de entrada para auxiliar um sistema de rotas de transporte, haja vista que a exposição do agregado acarreta desgaste excessivo nos pneus, maior consumo de combustível e disposição para um maior ruído e desconforto ao rolamento. Essas características possuem significativa importância na avaliação funcional dos pavimentos.

Destaca-se, também, o alto rendimento do Roadroid para o levantamento de campo e o processamento dos dados, principalmente, quando associado a um Sistema de Informação Geográfica (SIG). Ao se comparar os aplicativos aos métodos tradicionais, o Roadroid tem desempenho superior com custo reduzido. 
A utilização do Roadroid juntamente com a análise dos dados fornecidos pode indicar a presença de defeitos superficiais. Os trechos que proporcionaram dispersão elevada de dados apresentaram maior quantidade de defeitos no revestimento, confirmados através de inspeções visuais feitas pelos autores. Os principais defeitos encontrados que contribuíram para a dispersão dos dados foram buracos e remendos, que geram maior desconforto ao rolamento.

Por fim, conclui-se que os smartphones se apresentam como uma alternativa viável na análise preliminar da condição funcional dos pavimentos, pois, através das informações obtidas e correta análise dos dados, pode auxiliar as tomadas de decisões dos órgãos gestores. Essas novas tecnologias desenvolvidas possuem baixo custo, fácil operação e alta produtividade com potencial de melhoria, podendo ser utilizadas em larga escala, ressaltando que uma tecnologia mais avançada não impede a utilização de outra mais tradicional.

\section{REFERÊNCIAS}

Alessandroni, G.; L. C. Klopfenstein; S. Delpriori; M. Dromedari; G. Luchetti; B. D. Paolini; A. Seraghiti; E. Lattanzi; V. Freschi; A. Carini e A. Boglioli (2014) Smartroadsense: Collaborative road surface condition monitoring. International Conference on Mobile Ubiquitous Computing, Systems, Services and Technologies, Ubicomm, p. 210-215. Disponível em: http://www.researchgate.net/profile/Emanuele Lattanzi/publication/271700011 SmartRoadSense Collaborative Road Surface Condition_Monitoring/links/54cf57c70cf24601c0933aaf.pdf. Acesso em 15/03/2017.

Aps, M. (2006) Classificação da Aderência pneu-pavimento pelo índice IFI - International Friction Index para revestimentos asfálticos. Tese (Doutorado em Engenharia de Transportes). Escola Politécnica da Universidade de São Paulo, São Paulo.

Bernucci, L. B.; L. M. G. Motta; J. A. P. Ceratti e J. B. Soares (2008) Pavimentação Asfáltica. Formação Básica para Engenheiros. Petrobras. Abeda. Rio de Janeiro.

Bisconsini, D. R (2016) Avaliação da irregularidade longitudinal dos pavimentos com dados coletados por smartphones. Dissertação (Mestrado em Infra-Estrutura de Transportes). Escola de Engenharia de São Carlos, Universidade de São Paulo. São Paulo.

Buttlar, W. G. e S. Islam (2014) Measurement of pavement roughness using an Android-based smartphone application. Transportation Research Board 93rd Annual Meeting, Washington D.C. DOI: 10.3141/2457-04.

Casa Civil (2012) Governo do Estado do Ceará. Disponível em: http://www.casacivil.ce.gov.br/index.php/noticias/5746-governo-do-estado-inaugura-o-trecho-quixeramobim-madalena (Acesso em: 01/03/2017)

CNT (2016) Relatório Gerencial: Pesquisa CNT de Rodovias 2016. 20ª ed. Confederação Nacional do Transporte. Brasília: CNT, SEST, SENAT.

DER-CE (2017) Sistema de Rotas e Trafegabilidade (SIRTRA). Departamento Estadual de Rodovias. Disponível em: http://mapas.der.ce.gov.br/ (Acesso em 02/03/2017)

DNER - Departamento Nacional de Estradas de Rodagem (1986) DNER - ES 173/86 - Método de nível e mira para calibração de sistemas medidores de irregularidade tipo resposta. Especificação de Serviço. Ministério dos Transportes, Rio de Janeiro.

DNIT - Departamento Nacional de Infraestrutura de Transportes (2013) Instrução de Serviço/DG No13. Ministério dos Transportes. Brasília, DF.

DNIT - Departamento Nacional de Infraestrutura de Transportes (2016) Condições das Rodovias. Ministério dos Transportes. Brasília, DF. Disponível em: http://servicos.dnit.gov.br/condicoes/ce.htm (Acesso em 12/03/2017)

Douangphachanh, V. e H. Oneyma (2014) Estimation of road roughness condition from smartphones under realistic settings. In: Proceedings of the 13th International Conference on ITS Telecommunications (ITST). 5-7. p. 433-439.

DOI:10.1109/ITST.2013.6685585. Disponível em: http://www.jwcn.eurasipjournals.com/content/pdf/1687-1499-2014114.pdf (Acesso em: 10/03/2017)

Eriksson, J.; L. Girod; B. Hull; R. Newton; S. Madden e H. Balakrishnan (2008) The pothole patrol: using a mobile sensor network for road surface monitoring. Proceedings of the 6th International Conference on Mobile Systems, Applications, and Services. New York - NY, pp. 29-39.

Forslof, L. (2014). Roughness and Texture. Página profissional do Linkedin (C, publicado em 30 de novembro de 2014. Disponível em: https://www.linkedin.com/pulse/20141130211746-97325448-roughness-and-texture/?trackingId=JYtbRki7vh46bmTSMRWW1g\%3D\%3D (Acesso em 10/03/2017)

Forslof, L. (2015). Roadroid: Continuous road condition monitoring with smart phones. Journal of Civil Engineering and Architecture, v. 9, p. 485-496. DOI: 10.17265/1934-7359/2015.04.012

González, A.; E. J. O'brien; Y. Y. LI e K. Cashell (2008) The use of vehicle acceleration measurements to estimate road roughness, Vehicle System Dynamics. International Journal of Vehicle Mechanics and Mobility, v. 46, n. 6, p. 483-499. DOI: $10.1080 / 00423110701485050$

Hanson, T. R. e C. Cameron (2012) Can a smartphone collect IRI data? Conference and Exhibition of the Transportation Association of Canada - Transportation: Innovations and Opportunities Fredection, NB, Canada. Disponível em: http://conf.tacatc.ca/english/annualconference/tac2012/docs/session29/hanson.pdf (Acesso em 11/03/2017) 
Hirpahuanca, D. L. (2016). Determinación y Comparación de la Regularidad Superficial del Pavimento de la Carretera CuscoUrcos, Usando Teléfonos Inteligentes y el Rugosímetro de MERLIN. Monografia (Graduação em Ingeniería Civil). Universidad Andina del Cusco - UANDINA.

Mohan, P.; V. N. Padmanabhan e R. N. Ramjee (2008) Rich monitoring of road and traffic conditions using mobile smartphones. In: 6th ACM conference on Embedded Network Sensor Systems, SenSys, ACM, New York, NY. p. 323-336

Oliveira, F. H. L.; L. C. Almeida e S. P. Ramos (2016). Estudo do comportamento da aderência e do conforto ao rolamento em pistas de pouso e decolagem. Transportes, v. 24, n.2. ISSN: 2237-1346(online). DOI:10.4237/transportes.v24.i2/1059.

Silva, P. F. A. (2005) Manual de patologia e manutenção de pavimentos. Ed. Pini. São Paulo.

Wang, W. e F. Guo (2016) RoadLab - Revamping Road Condition and Road Safety Monitoring by Crowdsourcing with Smartphone App. Transportation Research Board 95rd Annual Meeting, Washington D.C. 